\title{
A class of nonassociative algebras
}

\author{
Michel Goze *- Elisabeth Remm ${ }^{\dagger}$ \\ Université de Haute Alsace, F.S.T. \\ 4, rue des Frères Lumière - 68093 MULHOUSE - France
}

A large class of nonassociative algebras, out of the Lie algebras has been studied, often with geometrical structures and sometimes on fondamental spaces as the spaces of Hochschild cohomology. In this paper, we propose to consider all these nonassociative algebras as algebras defined by the action of invariant subspaces of the symmetric group $\Sigma_{3}$ on the associator of the considered laws.

\section{$1 \quad \mathcal{V}$-algebras and $v$-algebras}

\section{$1.1 \quad$ Notations}

Let $\Sigma_{3}$ be the symmetric group of order 3 and $\mathbb{K}\left[\Sigma_{3}\right]$ the associated group algebra, where $\mathbb{K}$ is a field of characteristic zero. Let $\tau_{i j}$ be the transposition echanging $i$ and $j, c_{1}=(1,2,3)$ and $c_{2}=c_{1}{ }^{2}$ the two 3-cycles of $\Sigma_{3}$. Every $v \in \mathbb{K}\left[\Sigma_{3}\right]$ is written

$$
v=a_{1} i d+a_{2} \tau_{12}+a_{3} \tau_{13}+a_{4} \tau_{23}+a_{5} c_{1}+a_{6} c_{2}
$$

or more generally

$$
v=\sum_{\sigma \in \Sigma_{3}} a_{\sigma} \sigma
$$

where $a_{\sigma} \in \mathbb{K}$.

Consider the natural right action of $\Sigma_{3}$ on $\mathbb{K}\left[\Sigma_{3}\right]$

$$
\begin{array}{llc}
\Sigma_{3} \times \mathbb{K}\left[\Sigma_{3}\right] & \rightarrow & \mathbb{K}\left[\Sigma_{3}\right] \\
\left(\sigma, \sum_{i} a_{i} \sigma_{i}\right) & \mapsto & \sum_{i} a_{i} \sigma^{-1} \circ \sigma_{i}
\end{array}
$$

For every $v \in \mathbb{K}\left[\Sigma_{3}\right]$ we note $\mathcal{O}(v)$ the corresponding orbit of $v$. Let $F_{v}=$ $\mathbb{K}(\mathcal{O}(v))$ be the linear subspace of $\mathbb{K}\left[\Sigma_{3}\right]$ generated by $\mathcal{O}(v)$. It is an invariant subspace of $\mathbb{K}\left[\Sigma_{3}\right]$. By Mashke's theorem it is a direct sum of irreducible invariant subspaces.

*corresponding author: e-mail: M.Goze@uha.fr

${ }^{\dagger}$ E.Remm@uha.fr. 


\section{$1.2 \quad \mathcal{V}$-algebras and $v$-algebras}

Let $(\mathcal{A}, \mu)$ be a $\mathbb{K}$-algebra where $\mu$ is the multiplication law of $\mathcal{A}$. We note by $A_{\mu}$ the associator of $\mu$ that is

$$
A_{\mu}=\mu \circ(\mu \otimes I d-I d \otimes \mu) .
$$

Every $\sigma \in \Sigma_{3}$ defines a linear map noted $\Phi_{\sigma}$ given by

$$
\begin{array}{cccc}
\Phi_{\sigma}: & \mathcal{A}^{\otimes^{3}} & \rightarrow & \mathcal{A}^{\otimes^{3}} \\
& x_{1} \otimes x_{2} \otimes x_{3} & \rightarrow & x_{\sigma^{-1}(1)} \otimes x_{\sigma^{-1}(2)} \otimes x_{\sigma^{-1}(3)} .
\end{array}
$$

If $v=\sum_{\sigma \in \Sigma_{3}} a_{\sigma} \sigma \in \mathbb{K}\left[\Sigma_{3}\right]$ we define the endomorphism of $\mathcal{A}^{\otimes^{3}}$ by putting:

$$
\Phi_{v}=\sum_{\sigma \in \Sigma_{3}} a_{\sigma} \Phi_{\sigma}
$$

Definition 1 An algebra $(A, \mu)$ is a $\mathcal{V}$-algebra if there exists $v \in \mathbb{K}\left[\Sigma_{3}\right]$ such that

$$
A_{\mu} \circ \Phi_{v}=0 .
$$

$A \mathcal{V}$-algebra is a v-algebra if for every $w \in \mathbb{K}\left[\Sigma_{3}\right]$ with $w \notin F_{v}$ we have that $A_{\mu} \circ \Phi_{v}=0$ and $A_{\mu} \circ \Phi_{w} \neq 0$

For a given vector $v$ in $\mathbb{K}\left[\Sigma_{3}\right]$ a vector $w \in F_{v}$ could exist such as $F_{w}$ is strictly contained in $F_{v}$. In this case a $v$-algebra is not a $w$-algebra. On the other hand the $w$-algbera is a $v$-algebra and for every vector $v^{\prime} \in F_{v}$ such that $F_{v^{\prime}}=F_{v}$, the notion of $v$ and $v^{\prime}$-algebra are similar.

Let us note that we have the following sequence for a $v$-algebra $(A, \mu)$

$$
\mathcal{A}^{\otimes^{3}} \stackrel{\Phi_{v}}{\longrightarrow} \mathcal{A}^{\otimes^{3}} \stackrel{A_{\mu}}{\longrightarrow} \mathcal{A} .
$$

which represent a short complex.

\section{Examples.}

1) Let $v=\sigma \in \Sigma_{3}$. In this case $A_{\mu} \circ \Phi_{v}=0$ is equivalent to $A_{\mu}=0$. This $v$-algebra is nothing other than an associative algebra.

2) Let $v=I d-\tau_{23}$. A $v$-algebra satisfies

$$
A_{\mu}\left(x_{1} \otimes x_{2} \otimes x_{3}-x_{1} \otimes x_{3} \otimes x_{2}\right)=0 .
$$

Such an algebra is a Pre-Lie algebra [4.

\section{$2 \quad$ Irreducible $v$-algebras}

A $v$-algebra will be call irreducible if the $\Sigma_{3}$-module $F_{v}$ is irreducible. In this case we have $\operatorname{dim} F_{v}=1$ or 2 . 


\subsection{One dimensional case}

Proposition 2 Let $v$ be in $\mathbb{K}\left[\Sigma_{3}\right]$ such that $\operatorname{dim} F_{v}=1$. Then $v$ is given, up to a multiplicative scalar, by one of the two vectors $V$ and $W$ with

$$
\begin{aligned}
& V=I d-\tau_{12}-\tau_{13}-\tau_{23}+c_{1}+c_{2}, \\
& W=I d+\tau_{12}+\tau_{13}+\tau_{23}+c_{1}+c_{2} .
\end{aligned}
$$

The first case correspond to the character of $\Sigma_{3}$ given by the signature, the second corresponding to the trivial case. We will keep the notation $V$ and $W$ to the end to designate these two found vectors. For a characterization of the corresponding $v$-algebras we have need to recall the following definition.

Definition $3 A \mathbb{K}$-algebra $\mathcal{A}$ with the multiplication $\mu$ is called a Lie-admissible if the bracket $[x, y]=\mu(x, y)-\mu(y, x)$ satisfies the Jacobi identities, that is the algebra $(\mathcal{A},[]$,$) is a Lie algebra.$

$A \mathbb{K}$-algebra $(\mathcal{A}, \mu)$ is called power-associative algebra if the associator $A_{\mu}$ satisfies

$$
A_{\mu}(x, x, x)=0 .
$$

The second condition $A_{\mu}(x, x, x)=0$ is equivalent to defining powers of a single element $x \in \mathcal{A}$ recursively by $x^{1}=x, x^{n+1}=\mu\left(x^{n}, x\right)$. An important class of power-associative algebra is the the class of Jordan algebras.

Theorem 4 There are two classes of irreducible v-algebras whose corresponding module $F_{v}$ is one-dimensional :

1. the Lie-admissible algebras given by $v=V$,

2. the power-associative algebras given by $v=W$.

Proof. Let $(\mathcal{A}, \mu)$ be a $V$-algebra. Then we have $A_{\mu} \circ \Phi_{V}=0$. If we develop we obtain:

$A_{\mu}(x, y, z)-A_{\mu}(y, x, z)-A_{\mu}(z, y, x)-A_{\mu}(x, z, y)+A_{\mu}(y, z, x)+A_{\mu}(z, x, y)=0$.

This condition is equivalent to say that $[x, y]=\mu(x, y)-\mu(y, x)$ satisfies the Jacobi identity:

$$
[x,[y, z]]+[y,[z, x]]+[z,[x, y]]=0 .
$$

Suppose now that $(\mathcal{A}, \mu)$ is a $W$-algebra. Then, putting $[x, y]=\mu(x, y)-\mu(y, x)$ and $\{x, y\}=\mu(x, y)+\mu(y, x)$, the condition $A_{\mu} \circ \Phi_{W}=0$ is equivalent to

$$
[x,\{y, z\}]+[y,\{z, x\}]+[z,\{x, y\}]=0 .
$$

This last condition is just the linearization of the relation

$$
A_{\mu}(x, x, x)=0 .
$$

In fact if $A_{\mu}(x, x, x)=0$, we have also $A_{\mu}(x+y, x+y, x+y)=0$ which gives $A_{\mu}(x, x, y)+A_{\mu}(x, y, x)+A_{\mu}(y, x, x)+A_{\mu}(x, y, y)+A_{\mu}(y, x, y)+A_{\mu}(y, y, x)=0$. 
Moreover $A_{\mu}(x+y+z, x+y+z, x+y+z)=0$ implies taken account of the previous relations:

$A_{\mu}(x, y, z)+A_{\mu}(x, z, y)+A_{\mu}(y, x, z)+A_{\mu}(y, z, x)+A_{\mu}(z, x, y)+A_{\mu}(z, y, x)=0$

and the algebra is a $W$-algebra. Then, if the characteristic of the field $\mathbb{K}$ is not 2 (in fact we suppose it equal to 0), the two last conditions are equivalent.

The classes of Lie-admissible algebras and power-associative algebras have been introduced by Albert in [1.

\subsection{Two dimensional case, $\mathbb{K}=\mathbb{R}$}

In this section we suppose that the $\mathbb{K}\left[\Sigma_{3}\right]$-module $F_{v}$ is irreducible and 2dimensional. It is clear that, in this case, $V$ nor $W$ do not belong to $F_{v}$.

Proposition 5 Let $v \in \mathbb{K}\left[\Sigma_{3}\right]$. If $F_{v}$ is a 2-dimensional irreducible invariant space generated by $v$ and $\tau_{12}(v)$ then

$$
\begin{aligned}
v & =a_{1} I d+a_{2} \tau_{12}+\left(\alpha a_{1}+\beta a_{2}\right) \tau_{13}-\left(\alpha a_{1}+(1+\beta) a_{2}\right) \tau_{23} \\
& +\left(\beta a_{1}+\alpha a_{2}\right) c_{1}-\left((1+\beta) a_{1}+\alpha a_{2}\right) c_{2},
\end{aligned}
$$

with $\alpha^{2}=1+\beta+\beta^{2}$.

Proof. Let $v=a_{1} I d+a_{2} \tau_{12}+a_{3} \tau_{13}+a_{4} \tau_{23}+a_{5} c_{1}+a_{6} c_{2}$ be in $\mathbb{K}\left[\Sigma_{3}\right]$ and suppose that $F_{v}$ is generated by $v$ and $\tau_{12}(v)$. As $\tau_{i j}(v) \in F_{v}$ the vector $v$ has to satisfied

$$
\sum_{i=1}^{6} a_{i}=0 .
$$

As $\left\{\tau_{1 i}\right\}_{i=2,3}$ generates $\Sigma_{3}$, the $\mathbb{K}\left[\Sigma_{3}\right]$-module $F_{v}$ is 2-dimensional if and only if $\left\{v, \tau_{12}(v)\right\}$ are independent and $\left\{v, \tau_{12}(v), \tau_{13}(v)\right\}$ are related. Let $\tau_{13}(v)=$ $\alpha v+\beta \tau_{12}(v)$ (let us recall that $\left.\sigma\left(\sum a_{i} \sigma_{i}\right)=\sum a_{i} \sigma^{-1} \circ \sigma_{i}\right)$. This is equivalent to the following system

$$
\left\{\begin{array}{l}
A a_{1}=B a_{2} ; A a_{3}=B a_{6} ; A a_{4}=B a_{5} \\
A a_{2}=B a_{1} ; A a_{6}=B a_{3} ; A a_{5}=B a_{4} \\
A=1-\alpha^{2}+\alpha^{2} \beta-\beta^{3} \\
B=\alpha-\alpha^{3}+\alpha \beta^{2}+\alpha \beta
\end{array}\right.
$$

which implies that $\left(A^{2}-B^{2}\right) a_{1}=\left(A^{2}-B^{2}\right) a_{2}=0$.

$1^{\text {srt }}$ case. $A^{2}-B^{2} \neq 0$. Then we have $a_{1}=a_{2}=0$. As $\tau_{i j}(v) \in F_{v}$ for $(i, j) \neq(1,2)$, we deduce $v=0$ which is impossible.

$2^{\text {nd }}$ case. $A^{2}-B^{2}=0$.

i) $A=B$ that is $1-\alpha^{2}+\alpha^{2} \beta-\beta^{3}=\alpha\left(1-\alpha^{2}+\beta^{2}+\beta\right)$. We deduce $A\left(a_{1}-a_{2}\right)=0$. Then if $A \neq 0$, we obtain $a_{1}=a_{2}, a_{3}=a_{6}, a_{4}=a_{5}$ and in this 
case $v=\tau_{12}(v)$ which contradicts the hypothesis. Thus $A=B=0$ and the coefficients $\alpha$ and $\beta$ satisfy

$$
\left\{\begin{array}{l}
1-\alpha^{2}+\alpha^{2}+\alpha^{2} \beta-\beta^{3}=0 \\
\alpha\left(1-\alpha^{2}+\beta^{2}+\beta\right)=0
\end{array}\right.
$$

If $\alpha=0, \beta=0$ (here we choose $\mathbb{K}=\mathbb{R}$ ), we have $W \in F_{v}$ and $F_{v}$ is not irreducible. Thus $\alpha \neq 0$ and

$$
\left\{\begin{array}{l}
1-\alpha^{2}=-\beta-\beta^{2} \\
1-\alpha^{2}=-\beta\left(\alpha^{2}-\beta^{2}\right)
\end{array}\right.
$$

If $\beta=0, \alpha=-1$ or $\alpha=1$.

Lemma 6 If $\beta=0$ and $\alpha=1$ then $v=\left(a_{1}, a_{2}, a_{1},-a_{1}-a_{2}, a_{2},-a_{1}-a_{2}\right)$.

Proof. We write in this case $\tau_{13}(v)=v$ which implies

$$
v=\left(a_{1}, a_{2}, a_{1}, a_{4}, a_{2}, a_{4}\right)
$$

As $\tau_{23}(v)=\alpha^{\prime} v+\beta^{\prime} \tau_{12}(v)$, we deduce that

$$
\left(\alpha^{\prime}-\beta^{\prime}\right)\left(a_{2}-a_{1}\right)=0 .
$$

If $a_{1}=a_{2}, v=\left(a_{1}, a_{1}, a_{1}, a_{4}, a_{1}, a_{4}\right)$ with

$$
\left\{\begin{array}{l}
a_{4}=\left(\alpha^{\prime}+\beta^{\prime}\right) a_{1} \\
a_{1}=\alpha^{\prime} a_{1}+\beta^{\prime} a_{4}=\alpha^{\prime} a_{4}+\beta^{\prime} a_{1}
\end{array}\right.
$$

which gives that $\beta^{\prime}=-1$ or $a_{4}=a_{1}$. The second case corresponds to the vecteur $W$. Thus we have

$$
v=\left(a_{1}, a_{1}, a_{1},-2 a_{1}, a_{1},-2 a_{1}\right)
$$

because $-2-\alpha^{\prime}+\alpha^{\prime 2}=0$ which means that $\alpha^{\prime}=-1$ or $\alpha^{\prime}=2$ but for this last choice $v=W$.

If $a_{1} \neq a_{2}$ then $\alpha^{\prime}=\beta^{\prime}$ and we deduce that

$$
\left\{\begin{array}{l}
a_{4}=\alpha^{\prime}\left(a_{1}+a_{2}\right) \\
a_{2}=\alpha^{\prime}\left(a_{1}+a_{4}\right) \\
a_{1}=\alpha^{\prime}\left(a_{2}+a_{4}\right)
\end{array}\right.
$$

which implies that $\left(a_{1}+a_{2}+a_{4}\right)\left(1-2 \alpha^{\prime}\right)=0$ and $a_{4}=-a_{1}-a_{2}$. The vector $v$ has the expecting form. If $\alpha^{\prime}=\frac{1}{2}$ then $v=W$. Whence the announced lemma.

Lemma 7 If $\beta=0$ and $\alpha=-1$ then $v=\left(a_{1}, a_{2},-a_{1}, a_{1}-a_{2},-a_{2},-a_{1}+a_{2}\right)$.

Proof. The calcul is similar to the previous proof.

Suppose now that $\beta \neq 0$. In this case the system between $\alpha$ and $\beta$ is equivalent to the single equation

$$
\alpha^{2}=1+\beta+\beta^{2} .
$$


We deduce $a_{3}=\alpha a_{1}+\beta a_{2}, a_{5}=\alpha a_{2}+\beta a_{1}$, and $\beta a_{6}=\left(1-\alpha^{2}\right) a_{1}-\alpha \beta a_{2}$. As $\alpha^{2}=1+\beta+\beta^{2}$ and $\beta \neq 0$, we deduce $a_{6}=-(1+\beta) a_{1}-\alpha a_{2}$. Likewise we have $a_{4}=-\alpha a_{1}-(1+\beta) a_{2}$. The vector $v$ has the following form :

$$
v=\left(a_{1}, a_{2}, \alpha a_{1}+\beta a_{2},-\alpha a_{1}-(1+\beta) a_{2}, \beta a_{1}+\alpha a_{2},-(1+\beta) a_{1}-\alpha a_{2}\right),
$$

with $\alpha^{2}=1+\beta+\beta^{2}$.

ii) $A=-B$. If $A \neq 0$, then $a_{1}=-a_{2}, a_{3}=-a_{6}$, and $a_{4}=-a_{5}$. This implies $v=\tau_{12}(v)$, which contradicts the hypothesis. Then $A=0$ and we come back in the previous case.

Consequence. Let $(A, \mu)$ be a $v$-algebra with $\operatorname{dim} F_{v}=2$ and $F_{v}$ irreducible. We have one of the following cases

$$
F_{v}=\mathbb{K}\{v, \tau(v)\} \text { or } F_{v}=\mathbb{K}\{v, c(v)\}
$$

where $\tau$ is a transposition and $c$ a 3-cycle. But all the transpositions are conjugated in $\Sigma_{3}$ then if $F_{v}=\mathbb{K}\{v, \tau(v)\}$ there exists $v^{\prime} \in F_{v}$ such that $F_{v^{\prime}}=F_{v}=\mathbb{K}\left\{v^{\prime}, \tau_{12}\left(v^{\prime}\right)\right\}$. In this case the expression of $v^{\prime}$ is given by the proposition 5. Now the second case $F_{v}=\mathbb{K}\{v, c(v)\}$ can be discussed. In fact as the transpositions generate $\Sigma_{3}$, the dependance of the vectors $\{v, \tau(v)\}$ for every tranposition implies the dependance of the vectors $\{v, c(v)\}$ for every 3 cycle. Then every $v$-algebra with $\operatorname{dim} F_{v}=2$ and $F_{v}$ irreducible is discribed by the vector $v^{\prime}$ such that $F_{v^{\prime}}=\mathbb{K}\left\{v^{\prime}, \tau_{12}\left(v^{\prime}\right)\right\}$. Thus we can take $v$ given by the propositon 5 . We have

$$
\begin{aligned}
v & =a_{1} I d+a_{2} \tau_{12}+\left(\alpha a_{1}+\beta a_{2}\right) \tau_{13}-\left(\alpha a_{1}+(1+\beta) a_{2}\right) \tau_{23} \\
& +\left(\beta a_{1}+\alpha a_{2}\right) c_{1}-\left((1+\beta) a_{1}+\alpha a_{2}\right) c_{2},
\end{aligned}
$$

with $\alpha^{2}=1+\beta+\beta^{2}$, and

$$
\begin{aligned}
\tau_{12}(v) & =a_{2} I d+a_{1} \tau_{12}-\left((1+\beta) a_{1}+\alpha a_{2}\right) \tau_{13}+\left(\beta a_{1}+\alpha a_{2}\right) \tau_{23} \\
& -\left(\alpha a_{1}+(1+\beta) a_{2}\right) c_{1}+\left(\alpha a_{1}+\beta a_{2}\right) c_{2} .
\end{aligned}
$$

Let us consider the vector $u=v-\tau_{12}(v)$. We have

$$
\begin{aligned}
\tau_{12}(u) & =-u \\
\tau_{13}(u) & =\tau_{13}(v)-c_{1}(v) \\
& =\alpha v+\beta \tau_{12}(v)-\beta(v)-\alpha \tau_{12} v=(\alpha-\beta)(u) \\
\tau_{23}(u) & =\tau_{23}(v)-c_{2}(v)=-\alpha v-(1+\beta) \tau_{12}(v)-(1+\beta) v-\alpha \tau_{12}(v) \\
& =-(\alpha+\beta+1)\left(v+\tau_{12}(v)\right)
\end{aligned}
$$

Thus $\left\{u, \tau_{23}(u)\right\}$ generate $F_{v}$ that is $F_{v}=F_{u}$ and every $v$-algebra is an $u$-algebra. But the vector $u$ as the simplified following form

$$
\begin{aligned}
u & =\left(a_{1}-a_{2}, a_{2}-a_{1},(\alpha+\beta+1) a_{1}+(\alpha+\beta) a_{2},-(\alpha+\beta) a_{1}-(\alpha+\beta+1) a_{2},\right. \\
& \left.(\alpha+\beta) a_{1}+(\alpha+\beta+1) a_{2},-(\alpha+\beta+1) a_{1}-(\alpha+\beta) a_{2}\right) \\
& =\left(\lambda_{1},-\lambda_{1}, \lambda_{2},-\lambda_{3}, \lambda_{3},-\lambda_{2}\right)
\end{aligned}
$$

with $\lambda_{1}+\lambda_{3}-\lambda_{2}=0$, that is

$$
u=\left(\lambda_{1},-\lambda_{1}, \lambda_{1}+\lambda_{3},-\lambda_{3}, \lambda_{3},-\lambda_{1}-\lambda_{3}\right) .
$$


Theorem 8 Every $v$-algebra with $F_{v}$ irreducible and 2-dimensional is given by the following identity:

$$
\begin{aligned}
& \lambda_{1}\left(A_{\mu}(x, y, z)-A_{\mu}(y, x, z)\right)+\left(\lambda_{1}+\lambda_{3}\right)\left(A_{\mu}(z, y, x)-A_{\mu}(z, x, y)\right. \\
& -\lambda_{3}\left(A_{\mu}(x, z, y)-A_{\mu}(y, z, x)\right)=0
\end{aligned}
$$

\section{$3 \quad$ Lie-admissible $\mathcal{V}$-algebras}

The aim of this section is to describe all the $v$-algebras which are Lie-admissible.

Lemma 9 A v-algebra is Lie-admissible if and only if $V \in F_{v}$.

Proof.

$(\Rightarrow)$ We have that

$$
\begin{aligned}
A_{\mu} \circ \Phi_{v}=0 & \Rightarrow A_{\mu} \circ \Phi_{\sigma(v)}=0 \text { for all } \sigma \in \Sigma_{3} \\
& \Rightarrow A_{\mu} \circ \Phi_{v^{\prime}}=0 \text { for all } v^{\prime} \in F_{v} .
\end{aligned}
$$

Thus we have that $V \in F_{v} \Rightarrow A_{\mu} \circ \Phi_{V}=0$ and the $v$-algebra is Lie-admissible. $(\Leftarrow)$ By definition of the $v$-algebra $A_{\mu} \circ \Phi_{v^{\prime}}=0$ if and only if $v^{\prime} \in F_{v}$ Then if the $v$-algebra is Lie-admissible the vector $V$ is in $F_{v}$.

Let us first recall some results on the particular Lie-admissible $v$-algebras which are the $G_{i}$-algebras.

\subsection{The $G_{i}$-algebras of $[7]$}

In [7] and [1] the class of Lie-admissible algebras related with the subgroups of $\Sigma_{3}$ has been classified. We recall briefly this result.

Definition 10 Let $G$ be a subgroup of $\Sigma_{3}$. We call $G$-associative algebra an algebra $(\mathcal{A}, \mu)$ satisfying

$$
\sum_{\sigma \in G} A_{\mu} \circ \Phi_{\sigma}=0
$$

We have denoted $G_{1}=I d, G_{2}=\left\{I d, \tau_{12}\right\}, G_{3}=\left\{I d, \tau_{23}\right\}, G_{4}=\left\{I d, \tau_{13}\right\}$, $G_{5}=\left\{I d, c_{1}, c_{2}\right\}, G_{6}=\Sigma_{3}$ the subgroups of $\Sigma_{3}$. When $G=G_{6}$ the class of $\Sigma_{3}$-associative algebras is the full class of Lie-admissible algebras, when $G=G_{1}$ the corresponding class is the full class of associative algebras. For other cases we obtain the class of Vinberg algebras for $G_{2}$, the pre-Lie algebras for $G_{3}$. Each one of these algebras can be presented as a $v$-algebra :

- the $\Sigma_{3}$-associative algebras are the $V$-algebras.

- the $\left(G_{1^{-}}\right)$associative algebras are the $v$-algebras with $v=I d$.

- the Vinberg algebras, i.e. the $G_{2}$-associative algebras are the $v$-algebras corresponding to $v=I d-\tau_{12}$.

- the pre-Lie algebras, i.e. the $G_{3}$-associative algebras are the $v$-algebras corresponding to $v=I d-\tau_{23}$. 
- the $G_{4}$-associative algebras are the $\left\{I d-\tau_{13}\right\}$-algebras.

- the $G_{5}$-associative algebras are the $\left\{I d+c_{1}+c_{2}\right\}$-algebras.

We will generalize this list considering not only the invariant spaces $F_{v}$ associated to a subgroup of $\Sigma_{3}$, but all the invariant spaces.

\subsection{Classification of Lie-admissible $\mathcal{V}$-algebras}

Theorem 11 Every Lie-admissible v-algebra corresponds to the following one:

- type (I): $\operatorname{dim} F_{v}=1$ and $\mu(x, y)=x . y$ satisfies:

$$
\begin{gathered}
(x \cdot y) . z-x \cdot(y \cdot z)-(y \cdot x) \cdot z+y \cdot(x \cdot z)-(x \cdot z) \cdot y+x \cdot(z \cdot y)-(z \cdot y) \cdot x+z \cdot(y \cdot x) \\
+(y . z) \cdot x-y \cdot(z \cdot x)+(z \cdot x) \cdot y-z \cdot(x \cdot y)=0 .
\end{gathered}
$$

This identity defines the category of Lie-admissible algebras.

- type (II): $\operatorname{dim} F_{v}=2$ and the v-algebra is a power-associative algebra given by

$$
(x \cdot y) \cdot z-x \cdot(y \cdot z)+(y \cdot z) \cdot x-y \cdot(z \cdot x)+(z \cdot x) \cdot y-z \cdot(x \cdot y)=0
$$

(that is a $G_{5}$-associative algebra).

- type (III): $\operatorname{dim} F_{v}=3$. The v-algebra corresponds to

$$
\begin{gathered}
\left(I I I_{1}\right)(x . y) \cdot z-x \cdot(y \cdot z)+t[(y \cdot x) \cdot z-y \cdot(x . z)]-[(x . z) \cdot y-x \cdot(z . y)] \\
-t[(z \cdot x) \cdot y-z \cdot(x \cdot y)]=0
\end{gathered}
$$

where $t \neq 1$ or by

$$
\left(I I I_{2}\right)(x \cdot y) \cdot z-x \cdot(y \cdot z)-(z \cdot y) \cdot x+z \cdot(y \cdot x) .
$$

or by

$$
\begin{gathered}
\left(I I I_{3}\right)(x \cdot y) \cdot z-x \cdot(y \cdot z)-(y \cdot x) \cdot z+y \cdot(x \cdot z)-2(x \cdot z) \cdot y+2 x \cdot(z \cdot y) \\
+2(y \cdot z) \cdot x-2 y \cdot(z \cdot x)=0 .
\end{gathered}
$$

- type (IV): $\operatorname{dim} F_{v}=4$. The v-algebras are of the following type.

$$
\begin{aligned}
& \left(I V_{1}\right) 2[(x \cdot y) \cdot z-x \cdot(y \cdot z)]+(1+t)[(y \cdot x) \cdot z-y \cdot(x \cdot z)]+(z \cdot y) \cdot x \\
& \quad-z \cdot(y \cdot x)+[(y \cdot z) \cdot x-y \cdot(z \cdot x)]+(1-t)[(z \cdot x) \cdot y-z \cdot(x \cdot y)]=0
\end{aligned}
$$

with $t \neq 1$,

$$
\begin{gathered}
\left(I V_{2}\right) 2[(x \cdot y) \cdot z-x \cdot(y \cdot z)]+[(y \cdot x) \cdot z-y \cdot(x \cdot z)]+[(x \cdot z) \cdot y-x \cdot(z \cdot y)] \\
+[(y \cdot z) \cdot x-y \cdot(z \cdot x)]+[(z \cdot x) \cdot y-z \cdot(x \cdot y)]=0 \\
\left(I V_{3}\right) 2[(x \cdot y) \cdot z-x \cdot(y \cdot z)]+[(z \cdot y) \cdot x-z \cdot(y \cdot x)]-[(x \cdot z) \cdot y-x \cdot(z \cdot y)] \\
+3[(y \cdot z) \cdot x-y \cdot(z \cdot x)]+[(z \cdot x) \cdot y-z \cdot(x \cdot y)]=0
\end{gathered}
$$


- type $(V): \operatorname{dim} F_{v}=5$ and we have

$$
\begin{gathered}
2[(x \cdot y) \cdot z-x \cdot(y \cdot z)]-(y \cdot x) \cdot z+y \cdot(x \cdot z)-(z \cdot y) \cdot x+z \cdot(y \cdot x) \\
-((x \cdot z) \cdot y+x \cdot(z \cdot y))+((y \cdot z) \cdot x-y \cdot(z \cdot x))=0
\end{gathered}
$$

- (type VI): $\operatorname{dim} F_{v}=6$. This correspond to the class of associative algebras

$$
(x . y) . z-x .(y . z)=0
$$

Proof.

1) $\operatorname{dim} F_{v}=1$. This corresponds to theorem 4.1.

2) $\operatorname{dim} F_{v}=2$. As $V \in F_{v}, F_{v}=F_{V} \oplus F_{W}$. We can take $v=\frac{1}{2}(V+W)$ that is $v=(1,0,0,0,1,1)$. Then $\mu$ satisfies

$$
A_{\mu}(x, y, z)+A_{\mu}(y, z, x)+A_{\mu}(z, x, y)=0 .
$$

3) $\operatorname{dim} F_{v}=3$. In this case $F_{v}=F_{V} \oplus F_{u}$ with $\operatorname{dim} F_{u}=2$ and $F_{u}$ irreducible because we have only two non equivalent irreducible 1-dimensional representations. We have seen that $u$ can be chosen of the form

$$
u=\left(\lambda_{1},-\lambda_{1}, \lambda_{1}+\lambda_{3},-\lambda_{3}, \lambda_{3},-\lambda_{1}-\lambda_{3}\right) .
$$

Suppose that $\lambda_{1} \neq 0$ and consider the vector $v^{\prime}=u-\lambda_{1} V$ :

$$
v^{\prime}=\left(0,0,2 \lambda_{1}+\lambda_{3}, \lambda_{1}-\lambda_{3},-\lambda_{1}+\lambda_{3},-2 \lambda_{1}-\lambda_{3}\right) .
$$

Then $\mathbb{K} \mathcal{O}\left(v^{\prime}\right)=\mathbb{K}\left\{v^{\prime}, \tau_{13}\left(v^{\prime}\right), \tau_{23}\left(v^{\prime}\right)\right\}$. This space is of dimension 3 if $(b \neq a)$ or $(a=-b \neq 0)$ with $a=2 \lambda_{1}+\lambda_{3}$ and $b=-\lambda_{1}+\lambda_{3}$. The first case correspond to $\lambda_{1} \neq 0$ and the second case to $\lambda_{1}=0, \lambda_{3} \neq 0$. If $\lambda_{1} \neq 0$, we can write

$$
v^{\prime}=(0,0, a,-b, b,-a)
$$

with $a \neq b$. To simplify, using $v^{\prime \prime}=\tau_{13}\left(v^{\prime}\right)$, we have $\mathbb{K} \mathcal{O}\left(v^{\prime \prime}\right)=\mathbb{K} \mathcal{O}\left(v^{\prime}\right)=$ $F_{v}$ and $v "=(a, b, 0,-a, 0,-b)$. If $a \neq 0$, divising by $a$ and putting $t=\frac{b}{a}$, thus we can come down to $v=(1, t, 0,-1,0,-t)$ with $t \neq 1(t=1$ corresponds to $\left.\lambda_{1}=0\right)$. This corresponds to the case $\left(I I I_{1}\right)$. If $a=0$ and $b \neq 0$, we have $v=(0,-1,0,0,0,1)$ and we obtain the case $\left(I I I_{2}\right)$. If $\lambda_{1}=0$, we get $u=\left(0,0, \lambda_{3},-\lambda_{3}, \lambda_{3},-\lambda_{3}\right)=\lambda_{3}(0,0,1,-1,1,-1)$. We take $u+V=$ $(1,-1,0,-2,2,0)$ and we find the case $\left(I I I_{3}\right)$.

4) $\operatorname{dim} F_{v}=4$. As $F_{V} \subset F_{v}, F_{v}=F_{u} \oplus F_{V} \oplus F_{W}$ with $\operatorname{dim} F_{u}=2$ and $F_{u}$ irreducible. The subspace $F_{u} \oplus F_{V}$ corresponds to the previous case. Let $v=(1, t, 0,-1,0,-t)$ a vector associated to an algebra of type $\left(I I I_{1}\right)$. In these conditions $t \neq 1$. Let $\widetilde{v}=v+W=(2,1+t, 1,0,1,1-t)$. Then $\operatorname{dim} F_{\widetilde{v}}=4$ (because $t \neq 1)$ and $W=\frac{1}{2}\left(\tau_{23}(\widetilde{v})+\widetilde{v}\right), V=(1-t)\left(-\frac{1}{2} \widetilde{v}-\frac{3}{2} \tau_{12}(\widetilde{v})+\tau_{13}(\widetilde{v})+\right.$ $\left.\tau_{23}(\widetilde{v})\right)$. Thus the $v$-algebras correspond to the equations

$$
\begin{aligned}
2 A_{\mu}(x, y, z)+ & (1+t) A_{\mu}(y, x, z)+A_{\mu}(z, y, x) \\
& +A_{\mu}(y, z, x)+(1-t) A_{\mu}(z, x, y)=0
\end{aligned}
$$


These algebras are Lie-admissible and power-associative.

If $v$ is generating an algebra of type $\left(I I I_{2}\right)$ then

$$
v=(1,0,-1,0,0,0)
$$

In this case $\widetilde{v}=v+W=(2,1,0,1,1,1)$.

Finally if $v=(1,-1,0,-2,2,0)$, the vector generating the algebras of type $\left(I V_{3}\right)$ is of type $\widetilde{v}=(2,0,1,-1,3,1)$.

5) $\operatorname{dim} F_{v}=5$. In this case $F_{v}=F_{v_{1}} \oplus F_{v_{2}} \oplus F_{V}$ with $F_{v_{1}}$ and $F_{v_{2}}$ irreducible and 2-dimensional. As $\operatorname{dim} F_{v}=5$, the vectors $v, \tau_{12}(v), \tau_{13}(v), \tau_{23}(v), c_{1}(v), c_{2}(v)$ are of rank 5. There exists a linear relation between these vectors. Putting $v=\alpha_{1} I d+\alpha_{2} \tau_{12}+\alpha_{3} \tau_{13}+\alpha_{4} \tau_{23}+\alpha_{5} c_{1}+\alpha_{6} c_{2}$, we have obviously

$$
v+\tau_{12}(v)+\tau_{13}(v)+\tau_{23}(v)+c_{1}(v)+c_{2}(v)=\sum_{i=1}^{6} \alpha_{i} W .
$$

But $W \notin F_{v}$ then $\sum_{i=1}^{6} \alpha_{i}=0$. We find again the characterization of invariant subspace of $\mathbb{K}\left[\Sigma_{3}\right]$ of codimension one:

"If $\operatorname{dim} F_{v}=5$ then $v$ satisfies $\sum \alpha_{i}=0 . "$

Then there exists only one type of such subspace. A basis of $F_{v}$ can be obtained by the vectors $\left\{e_{1}=(1,0,0,0,0,-1), e_{2}=(0,1,0,0,0,-1), e_{3}=(0,0,1,0,0,-1)\right.$, $\left.e_{4}=(0,0,0,1,0,-1), e_{5}=(0,0,0,0,1,1)\right\}$.

Consider for example the vector $e_{1}$ and let us compute $\mathcal{O}\left(e_{1}\right)$. We obtain

$$
\mathcal{O}\left(e_{1}\right)=\left\{e_{1}, \tau_{12}\left(e_{1}\right), \tau_{13}\left(e_{1}\right), c_{1}\left(e_{1}\right)\right\}
$$

and $\operatorname{dim} \mathbb{K}\left(\mathcal{O}\left(e_{1}\right)\right)=4$. But $V \notin \mathbb{K}\left(\mathcal{O}\left(e_{1}\right)\right)$ and $\operatorname{dim} \mathbb{K}\left(\mathcal{O}\left(e_{1}+V\right)\right)=5$. The unicity of $F_{v}$ implies that we can reduce the vector $v$ to $e_{1}+V$. Then the class of Lie-admissible $v$-algebras with $\operatorname{dim} F_{v}=5$ is given by $v=(2,-1,-1,-1,1,0)$ that is it satisfies

$$
\begin{aligned}
(V): \quad & 2 x \cdot(y \cdot z)-2(x \cdot y) \cdot z-y \cdot(x \cdot z)+(y \cdot x) \cdot z-z \cdot(y \cdot x)+(z \cdot y) \cdot x \\
& -x \cdot(z \cdot y)+(x \cdot z) \cdot y+y \cdot(z \cdot x)-(y \cdot z) \cdot x=0 .
\end{aligned}
$$

6) $\operatorname{dim} F_{v}=6:$ If $\operatorname{dim} F_{v}=6$ then $F_{v}=\mathbb{K}\left[\Sigma_{3}\right]$. Let us consider the vector $e_{1}=(1,0,0,0,0,0)$. We have $\mathbb{K}\left(\mathcal{O}\left(e_{1}\right)\right)=\mathbb{K}\left[\Sigma_{3}\right]=F_{v}$. Then $v$ can be reduced to the vector $e_{1}$. In this case a Lie-admissible $v$-algebra satisfies $x .(y . z)-(x . y) . z=0$ that is, we find again the class of associative algebras.

Remark. The correspondance between the $v$-algebras and the $G_{i}$-associative algebras is the following: type $(I)$ corresponds to $G_{6}$, type $(I I)$ to $G_{5}$, type $\left(I I I_{1}\right)$ to $G_{2}$ when $t=-1$ and to $G_{3}$ when $t=0$, type $\left(I I I_{2}\right)$ corresponds to $G_{4}$. 


\section{Power-associative $v$-algebras}

\subsection{Classification}

We have seen that the category of power-associative algebras corresponds to the class of the $W$-algebras. In the previous section some classes of Lie-admissible algebras are also power-associative algebras. These cases correspond to $v$-algebras which $F_{v}$ contains $F_{V} \oplus F_{W}$. This appears if

- $\operatorname{dim} F_{v}=2: F_{v}=F_{V} \oplus F_{W}$;

- $\operatorname{dim} F_{v}=4: F_{v}=F_{u} \oplus F_{V} \oplus F_{W}$;

- $\operatorname{dim} F_{v}=6: F_{v}=\mathbb{K}\left[\Sigma_{3}\right]$.

Then it remains only the cases $\operatorname{dim} F_{v}=3$ and $\operatorname{dim} F_{v}=5$.

First case. We have $\operatorname{dim} F_{v}=3$ and $F_{v}=F_{u} \oplus F_{W}$ with $F_{u}$ irreducible and 2-dimensional. The vector $u$ is of the form $u=\left(\lambda_{1},-\lambda_{1}, \lambda_{1}+\lambda_{3},-\lambda_{3}, \lambda_{3},-\lambda_{1}-\right.$ $\left.\lambda_{3}\right)$. If $\lambda_{1} \neq 0$, then $v=u-\lambda_{1} W$ satisfies $\operatorname{dim} \mathbb{K}(\mathcal{O}(v))=3$ as soon as $\lambda_{1} \neq 0$ or $\lambda_{1}+\lambda_{3} \neq 0$. If these conditions are satisfied, $\widetilde{v}=(0,-2, t,-1-t,-1+t,-2-t)$ generates $\mathbb{K}(\mathcal{O}(v))$.

If $\lambda_{1}=0$ then $u=\left(0,0, \lambda_{3},-\lambda_{3}, \lambda_{3},-\lambda_{3}\right)$ and $\widetilde{v}=\frac{u}{\lambda_{3}}+W=(1,1,1,0,1,0)$ generates $F_{v}$.

Second case. $\operatorname{dim} F_{v}=5$. Then $F_{v}=F_{u_{1}} \oplus F_{u_{2}} \oplus F_{W}$ with $F_{u_{i}}$ irreducible and 2-dimensional for $i=1,2$. If $v=\left(a_{1}, a_{2}, a_{3}, a_{4}, a_{5}, a_{6}\right)$ then

$$
v-\tau_{12}(v)-\tau_{13}(v)-\tau_{23}(v)+c_{1}(v)+c_{2}(v)=\left(a_{1}-a_{2}-a_{3}-a_{4}+a_{5}+a_{6}\right) V .
$$

As $V \notin F_{v}$, we have that $a_{1}-a_{2}-a_{3}-a_{4}+a_{5}+a_{6}=0$ and

$$
F_{v}=\left\{\left(a_{1}, a_{2}, a_{3}, a_{4}, a_{5}, a_{6}\right) / a_{1}-a_{2}-a_{3}-a_{4}+a_{5}+a_{6}=0\right\} .
$$

Let $v_{1}=(1,0,0,0,0,-1)$ be in $F_{v}$. We have proved in the last section that $\mathbb{K}\left(\mathcal{O}\left(v_{1}\right)\right)$ is of dimension 4 . Then $v=v_{1}+W$ is a "generator" of $F_{v}$. As $v=(2,1,1,1,1,0)$, te corresponding power-associative $v$-algebra satisfies

$$
2 A_{\mu}(x, y, z)+A_{\mu}(y, x, z)+A_{\mu}(z, y, x)+A_{\mu}(x, z, y)+A_{\mu}(y, z, x)=0 .
$$

Theorem 12 Every power-associative v-algebra corresponds to the following one:

- type $\left(I^{\prime}\right): \operatorname{dim} F_{v}=1$ and $\mu(x, y)=x . y$ satisfies:

$(x . y) \cdot z-x \cdot(y \cdot z)+(y \cdot x) \cdot z-y \cdot(x . z)+(x . z) \cdot y-x \cdot(z \cdot y)+(z \cdot y) \cdot x-z \cdot(y \cdot x)$ $+(y \cdot z) \cdot x-y \cdot(z \cdot x)+(z \cdot x) \cdot y-z \cdot(x \cdot y)=0$.

This identity defines the category of power-associative algebras.

- type $(I I)\left(\operatorname{dim} F_{v}=2\right)$ and we have the algebras defined in theorem 11.

- type $\left(I I I^{\prime}\right)$ : $\operatorname{dim} F_{v}=3$. The v-algebra correspond to

$\left(I I I^{\prime}{ }_{1}\right)$ :

$$
\begin{gathered}
-2[(x \cdot y) . z-x \cdot(y \cdot z)]-(2+t)[(z \cdot y) \cdot x-z \cdot(y \cdot x)]+(t-1)[(x \cdot z) \cdot y-x \cdot(z \cdot y)] \\
-(1+t)[(y \cdot z) \cdot x-y \cdot(z \cdot x)]+t[(z \cdot x) \cdot y-z \cdot(x \cdot y)]=0,
\end{gathered}
$$


$\left(I I I^{\prime}{ }_{2}\right):$

$(x \cdot y) \cdot z-x \cdot(y \cdot z)+(y \cdot x) \cdot z-y \cdot(x \cdot z)+(z \cdot y) \cdot x-z \cdot(y \cdot x)+(y \cdot z) \cdot x-y \cdot(z \cdot x)$.

- type $(I V): \operatorname{dim} F_{v}=4$. The v-algebras are of type $\left(I V_{1}\right)$ or $\left(I V_{1}\right)$ of the theorem 11.

- type $\left(V^{\prime}\right): \operatorname{dim} F_{v}=5$ and we have

$$
\begin{gathered}
2[(x \cdot y) \cdot z-x \cdot(y \cdot z)]+(y \cdot x) \cdot z-y \cdot(x \cdot z)+(z \cdot y) \cdot x-z \cdot(y \cdot x) \\
+((x \cdot z) \cdot y-x \cdot(z \cdot y))+((y \cdot z) \cdot x-y \cdot(z \cdot x))=0
\end{gathered}
$$

This class corresponds to the alternative algebras.

- type $(V I): \operatorname{dim} F_{v}=6$ and we have the class of associative algebras.

Let us examine more paticularly type $\left(V^{\prime}\right)$.

Definition 13 An algebra $(\mathcal{A}, \mu)$ is alternative if its product satisfies

$$
A_{\mu}(x, x, y)=A_{\mu}(y, x, x)=0
$$

This condition is equivalent to the following system

$$
A_{\mu} \circ \Phi_{v_{1}}=A_{\mu} \circ \Phi_{v_{2}}=A_{\mu} \circ \Phi_{v_{3}}=0
$$

with $v_{1}=I d+\tau_{12}, v_{2}=I d-c_{1}, v_{3}=I d+\tau_{13}$. The vectors are in the orbit of the vector $v$ associated to the type $\left(V^{\prime}\right)$ and in fact $\mathbb{K}\left(\mathcal{O}\left\{v_{1}, v_{2}, v_{3}\right\}\right)=\mathcal{K}(\mathcal{O}(v))$. So the calss of algebras of type $\left(V^{\prime}\right)$ corresponds to the class of alternative algebras.

\subsection{A characteristic example: the octonions algebra}

The octonions algebra also called Caley algebra is a 8-dimensional algebra which is a $W$-algebra that is a power-associative $\mathcal{W}$-algebra of type $\left(V^{\prime}\right)$. More generally the 8-dimensional Caley-Dixon algebras of composition are $W$-algebras of type $V^{\prime}$.

Recall that any division composition algebra over $\mathbb{R}$ or $\mathbb{C}$ or the quaternions's algebras or the octonions's algebras.

\section{$5 \quad$ Tensor products of $\mathcal{V}$-algebras}

It is wellknown that the category of associative algebras is stable by tensor product. We saw in [7] that it is not the case for the $G_{i}$-associative algebras which are not associative. Here we will show that

Proposition 14 Let $A$ and $B$ be two $\mathcal{V}$-algebras. Then $A \otimes_{\mathbb{K}} B$ is a $\mathcal{V}$-algebra if and only $A$ and $B$ are associative. 
Proof. Let $A_{\mu}$ the associator of the law $\mu$ and $A_{\mu}^{L}\left(x_{1} \otimes x_{2} \otimes x_{3}\right)=\mu\left(\mu\left(x_{1}, x_{2}\right), x_{3}\right)$ and $A_{\mu}^{R}=A_{\mu}^{L}-A_{\mu}$. As $A$ (resp. $B$ ) is a $\mathcal{V}$-algebra, there exits $v \in \mathbb{K}$ (resp. $w \in \mathbb{K}$ ) such that $A_{\mu_{A}} \circ \Phi_{v}=0$ (resp. $A_{\mu_{B}} \circ \Phi_{w}=0$ ). Let us suppose that $A \otimes B$ is a $\mathcal{V}$-algebra. There exists $v^{\prime} \in \mathbb{K}$ such that $\left.A_{\mu_{A \otimes B}} \circ \Phi_{v^{\prime}}=0\right)$. By taking $v^{\prime}=\sum_{i=1}^{6} \gamma_{i} \sigma_{i}$ the last condition can be written

$$
\sum_{i=1}^{6} \gamma_{i}\left[A_{\mu_{A \otimes B}} \circ \Phi_{\sigma_{i}}\right]=0
$$

which can also be written

$$
\sum_{i=1}^{6} \gamma_{i}\left[A_{\mu_{A}}^{L} \circ \Phi_{\sigma_{i}} \otimes A_{\mu_{B}}^{L} \circ \Phi_{\sigma_{i}}-A_{\mu_{A}}^{R} \circ \Phi_{\sigma_{i}} \otimes A_{\mu_{B}}^{R} \circ \Phi_{\sigma_{i}}\right]=0 .
$$

Let us denote $e_{i}=A_{\mu_{A}}^{L} \circ \Phi_{\sigma_{i}}, \widetilde{e}_{i}=A_{\mu_{A}}^{R} \circ \Phi_{\sigma_{i}}, f_{i}=A_{\mu_{B}}^{L} \circ \Phi_{\sigma_{i}}$ and $\widetilde{f}_{i}=A_{\mu_{B}}^{R} \circ \Phi_{\sigma_{i}}$. The vectors $e_{i}$ and $\widetilde{e}_{i}$ belongs to $\operatorname{Hom}\left(A^{\otimes^{3}}, A\right)$ and the vectors $f_{i}$ and $\widetilde{f}_{i}$ to $\operatorname{Hom}\left(B^{\otimes^{3}}, B\right)$. The previous equation becomes:

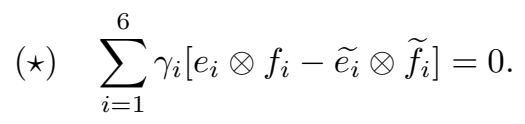

From the definition of the algebras $A$ and $B$ we have:

$$
\sum_{i=1}^{6} a_{i}\left(e_{i}-\widetilde{e}_{i}\right)=0 \text { and } \sum_{i=1}^{6} b_{i}\left(f_{i}-\widetilde{f}_{i}\right)=0
$$

if $v=\sum_{i=1}^{6} a_{i} \sigma_{i}$ and $w=\sum_{i=1}^{6} b_{i} \sigma_{i}$. Suppose that $\operatorname{dim}_{w}=k$ with $0 \leq k \leq 6$. Then the rank of the vectors $\left\{f_{i}, \tilde{f}_{i}\right\}$ is equal to $(6+k)$. We can suppose that $\left\{f_{1}, \ldots, f_{6}, \widetilde{f}_{1}, \ldots, \widetilde{f}_{k}\right\}$ are independent. This implies:

$$
\left\{\begin{array}{l}
\widetilde{f}_{k+1}=\rho_{1}^{k+1} f_{1}+\ldots+\rho_{6}^{k+1} f_{6}+\widetilde{\rho}_{1}^{k+1} \widetilde{f}_{1}+\ldots+\widetilde{\rho}_{k}^{k+1} \widetilde{f}_{k} \\
: \\
: \\
\widetilde{f}_{6}=\rho_{1}^{6} f_{1}+\ldots+\rho_{6}^{6} f_{6}+\widetilde{\rho}_{1}^{6} \widetilde{f}_{1}+\ldots+\widetilde{\rho}_{k}^{6} \widetilde{f}_{k} .
\end{array}\right.
$$

The equation $(\star)$ is then written:

$$
\sum_{i=1}^{6} e_{i}^{\prime} \otimes f_{i}+\sum_{i=1}^{k} e_{i} \otimes \widetilde{f}_{k}=0
$$


and the independence of the vectors $\left\{f_{1}, \ldots, f_{6}, \widetilde{f}_{1}, \ldots, \widetilde{f}_{k}\right\}$ implies $e_{1}^{\prime}=\ldots=$ $e_{6}^{\prime}=e^{\prime}{ }_{1}=\ldots=e^{\prime \prime}{ }_{k}=0$. Then we deduce

$$
\left\{\begin{array}{l}
\gamma_{1} e_{1}-\gamma_{k+1} \rho_{1}^{k+1} \widetilde{e}_{1}-\ldots-\gamma_{6} \rho_{1}^{6} \widetilde{e}_{6}=0 \\
\vdots \\
\gamma_{6} e_{6}-\gamma_{k+1} \rho_{6}^{k+1} \widetilde{e}_{1}-\ldots-\gamma_{6} \rho_{6}^{6} \widetilde{e}_{6}=0 \\
\gamma_{1} \widetilde{e}_{1}+\gamma_{k+1} \widetilde{\rho}_{1}^{k+1} \widetilde{e}_{k+1}+\ldots+\gamma_{6} \widetilde{\rho}_{1}^{6} \widetilde{e}_{6}=0 \\
\vdots \\
\vdots \\
\gamma_{k} \widetilde{e}_{k}+\gamma_{k+1} \widetilde{\rho}_{k}^{k+1} \widetilde{e}_{k+1}+\ldots+\gamma_{6} \widetilde{\rho}_{k}^{6} \widetilde{e}_{6}=0
\end{array}\right.
$$

Let us suppose that $k \neq 0$. Then the conditions on the vector $v$ are of the type $\sum_{i=1}^{6} a_{i}\left(e_{i}-\widetilde{e}_{i}\right)=0$ and we have succesively $\gamma_{1}=\ldots=\gamma_{k}=0$ and $\gamma_{k+1} \widetilde{\rho}_{i}^{k+1}=\ldots=\gamma_{6} \widetilde{\rho}_{i}^{6}=0$ for $i=1, \ldots, 6$. If one of $\gamma_{j}$ for $j=k+1, \ldots, 6$ is not 0 then $\widetilde{\rho}_{j}^{i}=0$ for $i=1, \ldots, 6$. Thus

$$
\tilde{f}_{j}=\rho_{1}^{j} f_{1}+\ldots+\rho_{6}^{j} f_{6}
$$

and, as for every $i$ there exists $\sigma \in \Sigma_{3}$ such that $\widetilde{f}_{i}=\widetilde{f}_{j} \circ \Phi_{\sigma}$, we have also

$$
\tilde{f}_{i}=\rho_{1}^{i} f_{1}+\ldots+\rho_{6}^{i} f_{6}
$$

for every $i=1, . ., 6$. This implies $k=0$ which is impossible from the hypothesis. Thus $k=0$ and the vectors $\left\{f_{i}, \widetilde{f}_{i}\right\}_{i=1, \ldots, 6}$ is of rank 6 . The only possible relations are then $f_{i}=\widetilde{f}_{i}$ and $B$ is associative. We deduce the associativity of $A$.

Remark. We have seen that a $W$-algebra is defined by the condition $A_{\mu}(x, x, x)=$ 0 for all $x$. Let $\mathcal{A}_{1}$ and $\mathcal{A}_{2}$ two $W$-algebras. For all $a \in \mathcal{A}_{1}$ and $b \in \mathcal{A}_{2}$ we have

$$
\begin{aligned}
& A_{\mu_{1} \otimes \mu_{2}}(a \otimes b, a \otimes b, a \otimes b)= \\
& =\mu_{1}\left(\mu_{1}(a, a), a\right) \otimes \mu_{2}\left(\mu_{2}(b, b), b\right)-\mu_{1}\left(a, \mu_{1}(a, a) \otimes \mu_{2}\left(b, \mu_{2}(b, b)\right)\right. \\
& =\left(\mu_{1}\left(\mu_{1}(a, a), a\right)-\mu_{1}\left(a, \mu_{1}(a, a)\right) \otimes \mu_{2}\left(\mu_{2}(b, b), b\right)\right. \\
& =0 .
\end{aligned}
$$

Then every indecomposable tensor in $A \otimes B$ generates an associative subalgebra. This doesnot imply, in general, that $A \otimes B$ is power-associative.

\section{A generalization : $\mathcal{V}-\mathcal{W}$-algebras}

The study of tensor products of $\mathcal{V}$-algebras has shown the necessity to write the associator as the difference

$$
A_{\mu}=A_{\mu}^{L}-A_{\mu}^{R}
$$

where $A_{\mu}^{L}\left(x_{1}, x_{2}, x_{3}\right)=\mu\left(\mu\left(x_{1}, x_{2}\right), x_{3}\right)$ and $A_{\mu}^{R}\left(x_{1}, x_{2}, x_{3}\right)=\mu\left(x_{1}, \mu\left(x_{2}, x_{3}\right)\right)$.

Now, instead of considering action of $\Sigma_{3}$-permutation on the associator we can consider it independently on $A_{\mu}^{L}$ and $A_{\mu}^{R}$ which will induce different symmetries. 


\subsection{Definition}

Definition 15 The catergory of $\mathcal{V}$ - $\mathcal{W}$-algebras correspond to the $\mathbb{K}$-algebras $(\mathcal{A}, \mu)$ for which exist $v, w \in \mathbb{K}\left[\Sigma_{3}\right]$ such that

$$
(\star)\left\{\begin{array} { l } 
{ A _ { \mu } ^ { L } \circ \Phi _ { v } = 0 } \\
{ A _ { \mu } ^ { R } \circ \Phi _ { w } = 0 }
\end{array} \quad \text { or } \quad ( \star \star ) \left\{A_{\mu}^{L} \circ \Phi_{v}-A_{\mu}^{R} \circ \Phi_{w}=0\right.\right.
$$

We will write that $(\mathcal{A}, \mu)$ is a $(v, w)$-algebra if $(\star)$ or $(\star \star)$ is satisfied and if for every $v^{\prime}, w^{\prime}$ such that $v \in F_{v^{\prime}}, w \in F_{w^{\prime}}$ and $v^{\prime} \notin F_{v}, w^{\prime} \notin F_{w}$ we have $A_{\mu}^{L} \circ \Phi_{v^{\prime}} \neq 0$ or $A_{\mu}^{R} \circ \Phi_{w^{\prime}} \neq 0$. It is clear that if $v=w$ or if $w \in F_{v}$ then a $(v, w)$ algebra is a $v$-algebra. Now the problem is to look if for a $(v, w)$-algebra there exists $v^{\prime}$ such that this algebra is a $v^{\prime}$-algebra. The most interesting example concerns the case of a $(v, w)$-algebra is a pre-Lie algebra or a $V$-algebra.

\subsection{Lie admissible $\mathcal{V}$ - $\mathcal{W}$-algebras}

Proposition 16 Let $(\mathcal{A}, \mu)$ be a $\mathcal{V}-\mathcal{W}$-algebras of type $(\star \star)$. Then if $\mathcal{A}$ is a Lie-admissible algebra we have :

1) $V \in F_{V} \cap F_{W}$

2) One of the two following conditions is satisfied:

a) $V=\sum a_{i} \sigma_{i}(v)=\sum a_{i} \sigma_{i}(w)$ i.e $\exists \chi \in \mathbb{K}\left[\Sigma_{3}\right]$ such that $\chi(v)=\chi(w)=V$,

b) there exits $\chi \in \mathbb{K}\left[\Sigma_{3}\right]$ such that $\chi(v-w)=0$ and $\chi(V) \neq 0$.

Proof. As the law $\mu$ satisfies

$$
A_{\mu}^{L} \circ \Phi_{v}-A_{\mu}^{R} \circ \Phi_{w}=0
$$

and if such a law is Lie-admissible then we have that $V \in F_{v}$ and $V \in F_{w}$. Let us first notice that to study $\mathcal{V}$ - $\mathcal{W}$-algebras is reduced to study the representations of $\Sigma_{3}$ in the vector space of $\widetilde{E}$ generated by $\left(x_{i} \cdot x_{j}\right) \cdot x_{k}$ and $x_{i} \cdot\left(x_{j} \cdot x_{k}\right)$ with $i, j, k \in\{1,2,3\}, i \neq j \neq k \neq i$. This vector space is 12-dimensional and isomorphic to $\mathbb{K}\left[\Sigma_{3}\right] \otimes \mathbb{K}\left[\Sigma_{3}\right]$. The invariant subvector spaces of $\widetilde{E}$ are also generated by a vector $\widetilde{v}$. When the algebra is a $\mathcal{V}$-algebra (considered as a $\mathcal{V}$ $\mathcal{W}$-algebra) we restrict the study to the representations of $\Sigma_{3}$ in $\widetilde{E}$ which let invariant the subspace $S=\left\{\left(x_{i} \cdot x_{j}\right) \cdot x_{k}-x_{i} \cdot\left(x_{j} \cdot x_{k}\right)\right\}$. In this case we have a representation with an interpretation as a representation of $\Sigma_{3}$ in $\mathbb{K}\left[\Sigma_{3}\right]$; this was the subject of the first part of this work.

Let us come back to

$$
A_{\mu}^{L} \circ \Phi_{v}-A_{\mu}^{R} \circ \Phi_{w}=0 .
$$

This relation can also be written

$$
A_{\mu}^{L} \circ \Phi_{v}-A_{\mu}^{R} \circ \Phi_{v}+A_{\mu}^{R} \circ \Phi_{v}-A_{\mu}^{R} \circ \Phi_{w}=0
$$

or

$$
A_{\mu} \circ \Phi_{v}-A_{\mu}^{R} \circ \Phi_{u}=0
$$


with $u=w-v$. As $V \in F_{v}$, if $\left\{\sigma_{1}(v)=v, \sigma_{2}(v), \ldots, \sigma_{k}(v)\right\}(k \leq 6)$, is a basis of $F_{v}$, then $V=\sum a_{i} \sigma_{i}(v)$ and

$$
A_{\mu} \circ \Phi_{V}=A_{\mu} \circ \Phi_{\sum a_{i} \sigma_{i}(v)}=\sum a_{i} A_{\mu} \circ \Phi_{\sigma_{i}(v)} .
$$

From the definition of $\Phi_{\sigma}$ as linear transformation of $\mathcal{A}^{\otimes^{3}}, \Phi_{\sigma(v)}=\Phi_{v} \circ \Phi_{\sigma^{-1}}$ which gives

$$
A_{\mu} \circ \Phi_{V}=\sum a_{i} A_{\mu} \circ \Phi_{v} \circ \Phi_{\sigma_{i}^{-1}}=A_{\mu} \circ \Phi_{v} \circ \Phi_{\sum a_{i} \sigma_{i}^{-1}} .
$$

We deduce

$$
A_{\mu} \circ \Phi_{V}-A_{\mu}^{R} \circ \Phi_{u} \circ \Phi_{\sum a_{i} \sigma_{i}^{-1}}=0 .
$$

Then

$$
A_{\mu} \circ \Phi_{V}-A_{\mu}^{R} \circ \Phi_{\sum a_{i} \sigma_{i}(u)}=0 .
$$

If the vector $u^{\prime}=\sum a_{i} \sigma_{i}(u)$ is not reduced to the identity, $\operatorname{dim} F_{u^{\prime}}<6$. If $F_{u^{\prime}}=0$, then $A_{\mu} \circ \Phi_{V}=0$ and the law $\mu$ is Lie-admissible. In this case $\sum a_{i} \sigma_{i}(u)=0$ that is $\sum a_{i} \sigma_{i}(v)=\sum a_{i} \sigma_{i}(w)=V$.

If $\operatorname{dim} F_{u^{\prime}} \neq 0$, as $\operatorname{dim} F_{u^{\prime}}<6$, the dual space is not trivial. Thus there exists $\chi^{\prime} \in \mathbb{K}\left[\Sigma_{3}\right]$ such that $\chi^{\prime}\left(u^{\prime}\right)=0$. The relation

$$
A_{\mu} \circ \Phi_{V}-A_{\mu}^{R} \circ \Phi_{u^{\prime}}=0
$$

implies

$$
A_{\mu} \circ \Phi_{\chi^{\prime}(V)}-A_{\mu}^{R} \circ \Phi_{\chi^{\prime}\left(u^{\prime}\right)}=0,
$$

from which

$$
A_{\mu} \circ \Phi_{\chi^{\prime}(V)}=0 .
$$

But $\chi^{\prime}(V)=a V$. If $a \neq 0$ then $A_{\mu} \circ \Phi_{V}=0$ and the $\mathcal{V}-\mathcal{W}$-algebra is Lieadmissible. Let us put $\chi^{\prime}=\sum c_{i} \sigma_{i}$. Then

$$
\chi^{\prime}\left(u^{\prime}\right)=\sum c_{i} \sigma_{i}\left(u^{\prime}\right)=\sum c_{i} a_{j} \sigma_{i}\left(\sigma_{j}(u)\right)=\sum c_{i} a_{j}\left(\sigma_{j} \circ \sigma_{i}\right)(u) .
$$

The vector $\chi=\sum c_{i} a_{j} \sigma_{j} \circ \sigma_{i}$ satisfies

$$
\chi(u)=0 .
$$

Corollary 17 If there exists $\chi^{\prime} \in \mathbb{K}\left[\Sigma_{3}\right]$ such that $\chi^{\prime}\left(u^{\prime}\right)=0$ and $\chi^{\prime}(V) \neq 0$ where $u^{\prime}=\sum_{i} \sigma_{i}(w-v)$ and $V=\sum a_{i} \sigma_{i}(v)$ then the $v-w$-algebra is Lieadmissible.

\section{Examples}

1. Consider the $(v, w)$-algebra defined by

$$
x .(y . z)-x .(z . y)-(x \cdot y) . z+(y \cdot x) . z=0
$$


Here $v=I d-\tau_{23}$ and $w=I d-\tau_{12}$. We have

$$
\begin{aligned}
& V=v-\tau_{12}(v)-\tau_{13}(v)=\sum a_{i} \sigma_{i}(v) \\
& V=w-\tau_{13}(w)-\tau_{23}(w)
\end{aligned}
$$

Then $V \in F_{v} \cap F_{w}$. The vector $u=(v-w)$ is written

$$
w-v=(0,-1,0,1,0,0)
$$

We have

$$
\sum a_{i} \sigma_{i}(u)=u-\tau_{12}(u)-\tau_{13}(u)=(1,-1,0,1,0,-1)=u^{\prime}
$$

Let $\chi^{\prime}=I d+\tau_{12}+\tau_{13}$. Then $\chi^{\prime}\left(u^{\prime}\right)=0$ with $\chi=I d+c_{1}+c_{2}$. This example plays a particular role in the study of Hopf operad. We will come back on this problem later.

2. Consider the $(v, w)$-algebra defined by

$$
2(x . y) . z-(y \cdot x) . z-(z . y) \cdot x-(x . z) \cdot y+(y . z) \cdot x-x .(y \cdot z)-y \cdot(z . x)-z .(x . y)
$$

Now $v=2 I d-\tau_{12}-\tau_{13}-\tau_{23}+c_{1}$ and $w=I d+c_{1}+c_{2}$. Here $\operatorname{dim} F_{v}=5$ $\operatorname{dim} F_{w}=2$. The vector $u=w-v=(-1,1,1,1,0,1)$ We have $V=w-\tau_{12}(w)$. Let us take $u^{\prime}=u-\tau_{12}(u)=(2,-2,0,-1,1,0)$. Then $\operatorname{dim} F_{u^{\prime}}=3$ and we have the relation $u^{\prime}+\tau_{12}\left(u^{\prime}\right)=\tau_{23}\left(u^{\prime}\right)+c_{1}\left(u^{\prime}\right)=\tau_{13}\left(u^{\prime}\right)+c_{2}\left(u^{\prime}\right)$. This implies that every $\chi^{\prime}$ such that $\chi^{\prime}\left(u^{\prime}\right)=0$ has the following form $\chi^{\prime}=\left(a_{1}, a_{1}, a_{3}, a_{2}, a_{2}, a_{3}\right)$. As $\chi^{\prime}(v)=0$ the $(v, w)$-algebra is not Lie-admissible. We can note that $u(V)$ is not zero.

\subsection{Monoidal category of $(\mathcal{V}, \mathcal{W})$-algebras}

Contrary to the case of $\mathcal{V}$-algebras where the tensor product works only with two associative algebras, we find by considering $(\mathcal{V}, \mathcal{W})$-algebras, new examples of categories stable by tensor product.

Proposition 18 Let $\mathcal{C}$ be the category of $(\mathcal{V}, \mathcal{W})$-algebras whose product satisfies:

$$
\mu\left(\mu\left(x_{1}, x_{2}\right), x_{3}\right)-\mu\left(x_{2}, \mu\left(x_{1}, x_{3}\right)\right)=0
$$

where $x_{1}, x_{2}, x_{3}$ are elements of the algebra. The category $\mathcal{C}$ is monoidal.

Proof. Let $A$ be an algebra. The algebra $A$ is a $(v, w)$-algebra by taking $v=I d$ and $w=c_{1}$.

Let $A$ and $B$ be two algebras of $\mathcal{C}$ and define the product of $A \otimes B$ :

$$
\forall a_{i} \in A, \forall b_{j} \in B, \quad\left(a_{1} \otimes b_{1}\right) \cdot_{A \otimes B}\left(a_{2} \otimes b_{2}\right)=\left(a_{1} \cdot{ }_{A} a_{2}\right) \otimes\left(b_{1} \cdot_{B} b_{2}\right)
$$

The product defined in this way eqquipes the tensor product of these algebras with a structure of algebra of the same type. In fact 


$$
\begin{aligned}
& \left.\left[\left(a_{1} \otimes b_{1}\right) \cdot\left(a_{2} \otimes b_{2}\right)\right] \cdot\left(a_{3} \otimes b_{3}\right)-\left(a_{2} \otimes b_{2}\right) \cdot\left[\left(a_{1} \otimes b_{1}\right)\right] \cdot\left(a_{3} \otimes b_{3}\right)\right] \\
& =\left(\left[a_{1} \cdot a_{2}\right] \cdot a_{3}\right) \otimes\left(\left[b_{1} \cdot b_{2}\right] \cdot b_{3}\right)-\left(a_{2} \cdot\left[a_{1} \cdot a_{3}\right]\right) \otimes\left(b_{2} \cdot\left[b_{1} \cdot b_{3}\right]\right) \\
& =\left(\left[a_{1} \cdot a_{2}\right] \cdot a_{3}\right) \otimes\left(\left[b_{1} \cdot b_{2}\right] \cdot b_{3}-b_{2} \cdot\left[b_{1} \cdot b_{3}\right]\right) \\
& =0
\end{aligned}
$$

Then $A \otimes B \in \mathcal{C}$.

\section{Associated operads}

As we have natural right action of $\Sigma_{3}$ we can define trivially the binary quadratic operad associated to each of these nonassociative type of algebras and their dual operads. We recall the basic definition of a binary quadratic operad and its dual operad before giving them for the nonassociative algebras defined below.

Let $\mathbb{K}\left[\Sigma_{n}\right]$ be the $\mathbb{K}$-group algebra of the symmetric group $\Sigma_{n}$. An operad $\mathcal{P}$ is defined by a sequence of $\mathbb{K}$-vector spaces $\mathcal{P}(n), n \geq 1$ such that $\mathcal{P}(n)$ is a right module over $\mathbb{K}\left[\sum_{n}\right]$ with composition maps

$$
\circ_{i}: \mathcal{P}(n) \otimes \mathcal{P}(m) \rightarrow \mathcal{P}(n+m-1) \quad i=1, \ldots, n
$$

satisfying some "associative" properties, the May Axioms ( 9 , 8]).

Any $\mathbb{K}\left[\Sigma_{2}\right]$-module $E$ generates a free operad noted $\mathcal{F}(E)$ satisfying $\mathcal{F}(E)(1)=$ $\mathbb{K}, \mathcal{F}(E)(2)=E$. In particular if $E=\mathbb{K}\left[\Sigma_{2}\right]$, the free module $\mathcal{F}(E)(n)$ admits as a basis the "parenthized products" of $n$ variables indexed by $\{1,2, \ldots, n\}$. For instance a basis of $\mathcal{F}(E)(2)$ is given by $\left(x_{1} . x_{2}\right)$ and $\left(x_{2} \cdot x_{1}\right)$, and a basis of $\mathcal{F}(E)(3)$ is given by

$$
\left\{\left(\left(x_{i} \cdot x_{j}\right) \cdot x_{k}\right),\left(x_{i} \cdot\left(x_{j} \cdot x_{k}\right) .\right), i \neq j \neq k \neq i, i, j, k \in\{1,2,3\}\right\} .
$$

Let $E$ be a $\mathbb{K}\left[\Sigma_{2}\right]$-module and $R$ a $\mathbb{K}\left[\Sigma_{3}\right]$-submodule of $\mathcal{F}(E)(3)$. We denote $\mathcal{R}$ the ideal generated by $R$, that is the intersection of all the ideals $\mathcal{I}$ of $\mathcal{F}(E)$ such that $\mathcal{I}(1)=0, \mathcal{I}(2)=0$ and $\mathcal{I}(3)=R$.

We call binary quadratic operad generated by $E$ and $R$ the operad $\mathcal{P}(\mathbb{K}, E, R)$, also denoted $\mathcal{F}(E) / \mathcal{R}$ and defined by

$$
\mathcal{P}(\mathbb{K}, E, R)(n)=(\mathcal{F}(E) / \mathcal{R})(n)=\frac{\mathcal{F}(E)(n)}{\mathcal{R}(n)}
$$

Thus an operad $\mathcal{P}$ is binary quadratic operad if and only if there exists a $\mathbb{K}\left[\Sigma_{2}\right]$ module $E$ and a $\mathbb{K}\left[\Sigma_{3}\right]$-submodule $R$ of $\mathcal{F}(E)(3)$ such that $\mathcal{P} \simeq \mathcal{F}(E) / \mathcal{R}$.

Examples.

1. The associative operad $\mathcal{A} s s$, the Lie operad $\mathcal{L} i e$ [5].

2. Let $\mathcal{F}(E)$ be the free operad generated by $E=\mathbb{K}\left[\Sigma_{2}\right]$. Consider the $\mathbb{K}\left[\Sigma_{3}\right]$-submodule $R$ generated by the vector

$$
\begin{aligned}
u= & x_{1} \cdot\left(x_{2} \cdot x_{3}\right)+x_{2} \cdot\left(x_{3} \cdot x_{1}\right)+x_{3} \cdot\left(x_{1} \cdot x_{2}\right)-x_{2} \cdot\left(x_{1} \cdot x_{3}\right)-x_{3} \cdot\left(x_{2} \cdot x_{1}\right) \\
& -x_{1} \cdot\left(x_{3} \cdot x_{2}\right)-\left(x_{1} \cdot x_{2}\right) \cdot x_{3}-\left(x_{2} \cdot x_{3}\right) \cdot x_{1}-\left(x_{3} \cdot x_{1}\right) \cdot x_{2}+\left(x_{2} \cdot x_{1}\right) \cdot x_{3} \\
& +\left(x_{3} \cdot x_{2}\right) \cdot x_{1}+\left(x_{1} \cdot x_{3}\right) \cdot x_{2}
\end{aligned}
$$


From now we will take

$$
E=\mathbb{K}\left[\Sigma_{2}\right]
$$

The Lie-Admissible operad, denoted $\mathcal{L} i e A d m$ is the binary quadratic operad defined by

$$
\mathcal{L} i e A d m=\mathcal{F}(E) / \mathcal{R} .
$$

For each of the above types of Lie-admissible and power-associative algebras there exists the corresponding operad. The way we define these algebras gives directely the vectors generating, as $\mathbb{K}\left[\Sigma_{3}\right]$-submodule, the module $R$ and thus the binary quadratic algebra associated to these algebras. Considering a $v$-Lieadmissible algebra, the corresponding module $F_{v}$ which determine the full class of this $v$-algebra, has a binary quadratic operad whose corresponding module $R$ is $F_{v}$. From the previous theorem, we know for each class of $v$-algebra the module of the associated operad. Then the relations of definition for all the operads of $v$-Lie-admissible algebras are given by this theorem.

\subsection{The dual operads associated to the Lie-admissible case}

Let us consider the binary quadratic operad $\mathcal{P}(\mathbb{K}, E, R)$. Then the dual binary quadratic operad is defined by

$$
\mathcal{P}^{!}=\mathcal{P}\left(\mathbb{K}, E^{\vee}, R^{\perp}\right)
$$

where $E^{\vee}$ is the dual of $E$ tensored by the signum representation of $\Sigma_{n}$ and $R^{\perp}$ the orthogonal complement to $R$ in $\mathcal{F}\left(E^{\vee}\right)(3)=\mathcal{F}(E)(3)^{\vee}$.

Proposition 19 The dual operads of algebras which are Lie-admissible are quadratic operads whose corresponding algebras are associative algebras satisfying respectively :

- for type $(I): x_{1} \cdot x_{2} \cdot x_{3}=x_{\sigma(1)} \cdot x_{\sigma(2)} \cdot x_{\sigma(3)}$ for all $\sigma \in \sigma_{3}$.

- for type $(I I): x_{1} \cdot x_{2} \cdot x_{3}=x_{2} \cdot x_{3} \cdot x_{1}=x_{3} \cdot x_{1} \cdot x_{2}$

- for type $\left(I I I_{1}\right): x_{1} \cdot x_{2} \cdot x_{3}+t x_{2} \cdot x_{1} \cdot x_{3}-x_{1} \cdot x_{3} \cdot x_{2}-t x_{3} \cdot x_{1} \cdot x_{2}=0$ with $t \neq 1$

- for type $\left(I I I_{2}\right): x_{1} \cdot x_{2} \cdot x_{3}=x_{3} \cdot x_{2} \cdot x_{1}$

- for type $\left(I I I_{3}\right): x_{1} \cdot x_{2} \cdot x_{3}-x_{2} \cdot x_{1} \cdot x_{3}-2 x_{1} \cdot x_{3} \cdot x_{2}+2 x_{2} \cdot x_{3} \cdot x_{1}=0$

-for type $\left(I V_{1}\right): t \neq 1$

$(t-1) x_{1} \cdot x_{2} \cdot x_{3}-(t-1) x_{2} \cdot x_{1} \cdot x_{3}-(t+2) x_{3} \cdot x_{2} \cdot x_{1}+(1+2 t) x_{1} \cdot x_{3} \cdot x_{2}$

$-(1+2 t) x_{2} \cdot x_{3} \cdot x_{1}+(t+2) x_{3} \cdot x_{1} \cdot x_{2}=0$

-for type $\left(I V_{2}\right): x_{1} \cdot x_{2} \cdot x_{3}+x_{2} \cdot x_{1} \cdot x_{3}-x_{3} \cdot x_{2} \cdot x_{1}-x_{3} \cdot x_{1} \cdot x_{2}=0$

-for type $\left(I V_{3}\right): x_{1} \cdot x_{2} \cdot x_{3}+x_{2} \cdot x_{1} \cdot x_{3}-x_{1} \cdot x_{3} \cdot x_{2}-x_{3} \cdot x_{1} \cdot x_{2}=0$

-for type $(V): x_{1} \cdot x_{2} \cdot x_{3}-x_{2} \cdot x_{1} \cdot x_{3}-x_{3} \cdot x_{2} \cdot x_{1}-x_{1} \cdot x_{3} \cdot x_{2}+x_{2} \cdot x_{3} \cdot x_{1}+x_{3} \cdot x_{1} \cdot x_{2}=0$ 
Proof. Let us consider the scalar product on $\mathcal{F}(E)(3)$ defined by

$$
\begin{aligned}
& <i(j k), i(j k)>=\operatorname{sgn}\left(\begin{array}{ccc}
1 & 2 & 3 \\
i & j & k
\end{array}\right) \\
& <(i j) k,(i j) k>=-\operatorname{sgn}\left(\begin{array}{ccc}
1 & 2 & 3 \\
i & j & k
\end{array}\right)
\end{aligned}
$$

Let $R$ be the $\mathbb{K}\left[\Sigma_{3}\right]$-submodule which determines the operad of algebras of type $(I)$. This corresponds to the Lie-admissible algebras or $G_{6}$-algebras in [7] hence the result.

If $R$ determines the operad of algebras of type $(I I)$ we get the $G_{5}$-algebras of [7.

Let $R$ be associated to one of the $\left(I I I_{i}\right)$ case. The annihilator $R^{\perp}$ respect to this scalar product is of dimension 9 . Let $R^{\prime}$ be the $\mathbb{K}\left[\Sigma_{3}\right]$-submodule of $\mathcal{F}(E)(3)$ generated by the vectors $\left(x_{1} x_{2}\right) x_{3}-x_{1}\left(x_{2} x_{3}\right)$ and

-for $\mathrm{i}=1\left(x_{1} \cdot x_{2}\right) \cdot x_{3}+t\left(x_{2} \cdot x_{1}\right) \cdot x_{3}-\left(x_{1} \cdot x_{3}\right) \cdot x_{2}-t\left(x_{2} \cdot x_{3}\right) \cdot x_{1}$ with $t \neq 1$;

-for $\mathrm{i}=2\left(x_{1} x_{2}\right) x_{3}-\left(x_{3} x_{2}\right) x_{1}$;

-for $\mathrm{i}=3\left(x_{1} \cdot x_{2}\right) \cdot x_{3}+\left(x_{3} \cdot x_{2}\right) \cdot x_{1}-\left(x_{2} \cdot x_{3}\right) \cdot x_{1}+\left(x_{2} \cdot x_{1}\right) \cdot x_{3}$.

Then $\operatorname{dim} R^{\prime}=9$ and $\langle u, v\rangle=0$ for all $v \in R^{\prime}$ where $u$ is the vector which generates $R$. This implies $R^{\prime} \simeq R^{\perp}$ and $(\mathcal{F}(E) / \mathcal{R})^{!}$is by definition the quadratic operad $\mathcal{F}(E) / \mathcal{R}^{\perp}$.

We demonstrate in the same way the case of $\left(I V_{i}\right)$ with $R^{\perp}$ is the $\mathbb{K}\left[\sum_{3}\right]$ sub-module of $\mathcal{F}(E)(3)$ generated by the vectors $\left(x_{1} x_{2}\right) x_{3}-x_{1}\left(x_{2} x_{3}\right)$ and

-for $\mathrm{i}=1(t-1)\left(x_{1} \cdot x_{2}\right) \cdot x_{3}-(t-1)\left(x_{2} \cdot x_{1}\right) \cdot x_{3}-(t+2)\left(x_{3} \cdot x_{2}\right) \cdot x_{1}+$

$(1+2 t)\left(x_{1} \cdot x_{3}\right) \cdot x_{2}-(1+2 t)\left(x_{2} \cdot x_{3}\right) \cdot x_{1}+(t+2)\left(x_{3} \cdot x_{1}\right) \cdot x_{2}$ with $t \neq 1$.

-for $\mathrm{i}=2\left(x_{1} \cdot x_{2}\right) \cdot x_{3}+\left(x_{2} \cdot x_{1}\right) \cdot x_{3}-\left(x_{3} \cdot x_{2}\right) \cdot x_{1}-\left(x_{3} \cdot x_{1}\right) \cdot x_{2}$;

-for $\mathrm{i}=3\left(x_{1} x_{2}\right) x_{3}+\left(x_{2} x_{1}\right) x_{3}-\left(x_{1} x_{3}\right) x_{2}-\left(x_{3} x_{1}\right) x_{2}$.

For the case $(V)$, the sub-module $R$ is of dimension 7 and generated as $\Sigma_{3}$-submodule by $\left(x_{1} x_{2}\right) x_{3}-x_{1}\left(x_{2} x_{3}\right)$ and $\left(x_{1} x_{2}\right) x_{3}-\left(x_{2} x_{1}\right) x_{3}-\left(x_{3} x_{2}\right) x_{1}-$ $\left(x_{1} x_{3}\right) x_{2}+\left(x_{2} x_{3}\right) x_{1}+\left(x_{3} x_{1}\right) x_{2}$.

\subsection{The dual operads associated to the power-associative case}

The determination of the dual operads in the power-associative case is similar to the Lie-admissible one. When $F_{v}$ is 2 or 4 -dimensional, as the $v$-algebras are both power-associative and Lie-admissible and the dual operads are described in the previous proposition.

Proposition 20 The dual operads of power-associative algebras are quadratic operads whose corresponding algebras are associative algebras satisfying respectively:

- for type $\left(I^{\prime}\right): x_{1} \cdot x_{2} \cdot x_{3}=-x_{2} \cdot x_{1} \cdot x_{3}=-x_{1} \cdot x_{3} \cdot x_{2}$

- for type $\left(I I I^{\prime}{ }_{1}\right)$ : 
$-2 x_{1} \cdot x_{2} \cdot x_{3}-(2+t) x_{3} \cdot x_{2} \cdot x_{1}+(t-1) x_{1} \cdot x_{3} \cdot x_{2}-(1+t) x_{2} \cdot x_{3} \cdot x_{1}+t x_{3} \cdot x_{1} \cdot x_{2}=0$

- for type $\left(I I I^{\prime}{ }_{2}\right): x_{1} \cdot x_{2} \cdot x_{3}+x_{2} \cdot x_{1} \cdot x_{3}+x_{3} \cdot x_{2} \cdot x_{1}+x_{2} \cdot x_{3} \cdot x_{1}=0$

- for type $\left(V^{\prime}\right): x_{1} \cdot x_{2} \cdot x_{3}+x_{2} \cdot x_{1} \cdot x_{3}+x_{3} \cdot x_{2} \cdot x_{1}+x_{1} \cdot x_{3} \cdot x_{2}+x_{2} \cdot x_{3} \cdot x_{1}+x_{3} \cdot x_{1} \cdot x_{2}=0$

When $F_{v}$ is 2 or 4-dimensional, as the v-algebras are both power-associative and Lie-admissible, we have define the dual operad in the previous proposition.

The proof is similar for the Lie-admissible and power-associative cases.

\subsection{An interpretation of algebras on the dual operad}

If we look the way to define the dual operad, we see that $\mathcal{P}^{!}=\frac{\mathcal{F}(E)}{\mathcal{R}^{\perp}}$ with $\mathcal{R}^{\perp}=<\left(x_{1} \cdot x_{2}\right) \cdot x_{3}-x_{1} \cdot\left(x_{2} \cdot x_{3}\right), u_{1}, \ldots, u_{p}>$. So $\mathcal{R}^{\perp}$ can be decomposed in two subspaces $R_{\text {ass }}$ which gives the associativity and a second one $U$ which gives the supplementary rules that an algebra on this operad has to satisfy.

Example. In the case of Vinberg algebras, we have

$$
R^{\perp}=R_{a s s} \oplus<\left(x_{1} \cdot x_{2}\right) \cdot x_{3}-\left(x_{2} \cdot x_{1}\right) \cdot x_{3}>.
$$

We can make such a decomposition so that every vector of $U$ is left parenthized and that $U$ is a $\mathbb{K}\left[\Sigma_{3}\right]$-module. Let us consider the $\mathbb{K}\left[\Sigma_{3}\right]$-module $\widetilde{E}=\left\{x_{i} \cdot x_{j} \cdot x_{k}, i \neq j \neq k \neq i\right\}$ of non parenthized 3-products, $\operatorname{dim} \widetilde{E}=6$. Then $U$ is isomorphic to a submodule $G$ of $\widetilde{E}$. There is a natural right action of $\mathbb{K}\left[\Sigma_{3}\right]$ on $\widetilde{E}$ :

$$
\begin{array}{ccc}
\widetilde{E} \times \mathbb{K}\left[\Sigma_{3}\right] & \rightarrow & \widetilde{E} \\
\left(x_{1} \cdot x_{2} \cdot x_{3}, \sigma\right) & \rightarrow & x_{\sigma^{-1}(1)} \cdot x_{\sigma^{-1}(2)} \cdot x_{\sigma^{-1}(3)}
\end{array}
$$

If we consider $A$ a $v$-algebra, the binary quadratic operad $\mathcal{P}$ associated to this algebra is such that $R=F_{v}$. We can naturally associated a sub-module $H$ of $\widetilde{E}$ to $F_{v}$ :

$$
\begin{array}{clc}
E & \rightarrow & \widetilde{E} \\
v=A_{\mu} \circ \sigma x_{1} \otimes x_{2} \otimes x_{3} & \rightarrow & \widetilde{v}=x_{\sigma^{-1}(1)} \cdot x_{\sigma^{-1}(2)} \cdot x_{\sigma^{-1}(3)}
\end{array}
$$

Then in $\widetilde{E}, \operatorname{dim} H=\operatorname{dim} F_{v}$ and $\operatorname{dim} G=\operatorname{codim} H=6-\operatorname{dim} H$.

We can also define a notion of $\widetilde{v}$-algebra in $\widetilde{E}$ that is $(A, \mu)$ is a $\widetilde{V}$-algebra if and only if $\Phi_{\widetilde{v}}=0$. We have similar results for the orbit of $\widetilde{v}$ under the previous action and decomposition of $\widetilde{\mathcal{O}}(\widetilde{v})$ in direct sum of irreducible invariant subspaces.

Let $V$ and $W$ be the vectors of $\mathbb{K}\left[\Sigma_{3}\right]$ corresponding to Lie-admissible and power-associative. As $\langle V, W\rangle \neq 0$, we have directely that if $V$ and $W$ are both in $F_{v}$, neither $\widetilde{V}$ nor $\widetilde{W}$ in $R^{\perp}$. Then $G$ is a direct sum of 2-dimensional irreducible invariant submodule of $E$. 
Proposition 21 Let $A$ be a v-algebra over an operad $\mathcal{P}=\frac{\mathcal{F}(E)}{\mathcal{R}}$.

- If $F_{v}$ contains $V$ and $W$ then $R^{\perp}$ defining the dual operad is

$$
R^{\perp}=R_{a s s} \oplus_{i} F_{w_{i}}
$$

where $F_{w_{i}}$ are 2-dimensional irreducible invariant submodules.

- If $F_{v}$ contains $V$ (resp. $W$ ) but not $W$ (resp. $V$ ) then

$$
R^{\perp}=R_{a s s} \oplus V \oplus_{i} F_{w_{i}}\left(\text { resp. } R_{a s s} \oplus W \oplus_{i} F_{w_{i}}\right)
$$

Example. If we consider a Lie-algebra of type $\left(I V_{1}\right)$ defined by $v=(t-1) I d-$ $(t-1) \tau_{12}-(t+2) \tau 13+(1+2 t) \tau 23-(1+2 t) c_{1}+(t+2) c_{2}, t \neq 1$, as $V$ and $W$ are in $F_{v}$, we can verify that $R^{\perp}=U \oplus F_{w_{1}}$ where $F_{w_{1}}$ is a 2-dimansional irreducible space.

\section{On the tensor product of algebras on operads}

We have seen in [7] that $G_{i}-A S S$ the category $G_{i}$-associative algebras on $\mathbb{K}$ for $i=2, \ldots, 6$ is not associated to a Hopf operad which implies that two algebras in the same category of $G_{i}$-algebras $(i \geq 6)$ do not give in general a $G_{i}$-algebra. But the functor $A \otimes-$ determines a one-to-one correspondence between the algebras over the operad $G_{i}-\mathcal{A} s s$ and algebras on the dual operad $G_{i}-\mathcal{A} s s^{!}$.

Theorem 22 (G.R) Let $A$ be a $G_{i}$-associative algebra. Then $A \otimes-$ is a covariant functor

$$
A \otimes-:\left(G_{i}-A S S\right)^{!} \longrightarrow G_{i}-A S S
$$

where $\left(G_{i}-A S S\right)$ ! is the category of algebras corresponding to the algebras over the dual operad $G_{i}-\mathcal{A} s s^{\text {! }}$.

Moreover, we can see that

Theorem 23 If $A$ is a $V$-algebra and $B$ a $W^{!}$-algebra (that is an algebra on the dual operad of the operad of $W$-algebras) then $A \otimes B$ can be provided with a product of $W$-algebra; if $A$ is a $W$-algebra and $B$ a $V^{!}$-algebra then $A \otimes B$ is a $W$-algebra; if $A$ is a $W$-algebra and $B$ a $W^{!}$-algebra then $A \otimes B$ is a $V$-algebra.

This shows a link between 1-dimensional $\mathcal{V}$ and $\mathcal{W}$-algebras and their "dual" algebras. In the case of 3 -dimensional $\mathcal{V}$ and $\mathcal{W}$-algebras, we have the same type of results that is if we call $\bar{G}_{i}-A s s$ and $\bar{G}_{i}-\mathcal{A} s s$ the corresponding category and operad associated to the $G_{i}-A s s$ and $G_{i}-\mathcal{A} s s$ in the power-associative case (that is $\bar{G}_{1}-$ Ass is the category of $W$-algebras, $\bar{G}_{2}-$ Ass is the category of $v$-algebras with $\left.v=I d+\tau_{12}, \ldots\right)$ we have that

Theorem 24 If $A$ is a $G_{i}-A s s$-algebra and $B$ a $\bar{G}_{i}^{!}-$Ass-algebra then $A \otimes B$ can be provided with a product of $G_{i}$-algebra; If $A$ is a $\bar{G}_{i}-$ Ass-algebra and $B$ a $G_{i}^{!}-$Ass-algebra then $A \otimes B$ is a $\bar{G}_{i}-$ Ass-algebra; If $A$ is a $\bar{G}_{i}$-algebra and $B$ a $\bar{G}_{i}^{!}$- Ass-algebra then $A \otimes B$ is a $\bar{G}_{i}-$ Ass-algebra; 
The description of operads in terms of $\Sigma_{3}$-invariant subspaces permits to generalize the case of $G_{i}$-algebras. What about the tensorial construction for the new Lie-admissible and power-associative algebras?

In the previous examples, what enables the construction is that we can factorize the fist part to make appear the fact that the algebra $A \otimes B$ is a $\mathcal{V}$ or $\mathcal{W}$-algebra. In fact, we have that $R \perp=R_{\text {ass }} \oplus U$ with $U$ isomorphic to $<I d+\varepsilon_{i} \sigma_{i}>$ with $\varepsilon_{i} \in\{-1,1\}$ and $\sigma_{i} \in \sigma_{3}, \sigma_{i} \neq I d$.

The possibilities for $U$ are $\left.\left.\left\langle I d-\tau_{12}\right\rangle,<I d-\tau_{13}\right\rangle,<I d-\tau_{23}\right\rangle$ which are of dimension 3 and correspond to the Vinberg, pre-Lie and $G_{5}$ case, $<I d+\tau_{12}>$ ,$<I d+\tau_{13}>,<I d+\tau_{23}>$ of dimension 3 and which are the corresponding power-associative cases, $\left\langle I d-c_{1}\right\rangle$ which is of dimension 4 and correspond to the dual operad of $\left.G_{5}\left(=\bar{G}_{5}\right),<I d-\tau_{12}, I d-c_{1}\right\rangle,<I d+\tau_{12}, I d-c_{1}>$ which are of dimension 5 and correspond respectively to the $G_{6}$ and $\bar{G}_{6}$ cases, $<I d+c_{1}>$ which is of dimension 6 and corresponds to the associative case $\left(<I d+c_{1}>=<I d>\right)$.

\section{References}

[1] Albert A.A., Power-associative rings. Trans. Amer. Math. Soc. 64 (1948), 552-593.

[2] Bremmer M., Hentzel I., Identities for the associator in altenative algebras. Preprint.

[3] Dzhumadil'daev A., Novikov-Jordan algebras. Comm. in Algebra 11, (2002), 5207-5240.

[4] Gerstenhaber M., The cohomology structure of an associative ring, Ann of math. 78, 2, (1963), 267-288.

[5] Ginzburg V., Kapranov M., Koszul duality for operads. Duke Math Journal. 76 1, (1994), 203-272.

[6] Elduque A. ,Myung H.C., Mutations of Alternative Algebras. Kluwer Academic Press. Math and its applications 278. 1994.

[7] Goze M., Remm E., Lie-admissible algebras and related operads. Preprint Mulhouse 2002.

[8] Markl M., Shnider S., Stasheff J., Operads in algebra, topology and physics. Mathematical Surveys and Monographs, 96. American Mathematical Society, Providence, RI, 2002. x+349 pp.

[9] Markl M. Distributive laws and Kozulness. Annales Institut Fourier. 46 , 2 , (1996), 307-323.

[10] Nijenhuis A., Sur une classe de propriétés communes à quelques types différents d'algèbres. Enseignement Math. 14 (2), (1970), 225-277. 
[11] Remm E., Opérades Lie-admissibles. C. R. Math. Acad. Sci. Paris 334 no. 12, (2002), 1047-1050.

[12] Schafer R.D. Introduction to Nonassociative Algebras. Academic Press. 1966 